\title{
A Method of Radar Echo Extrapolation Based on TREC and Barnes Filter
}

\author{
HAIBO ZOU \\ Meteorological Sciences Institute of Jiangxi Province, and Meteorological Disaster Emergency \\ Warning Center of Jiangxi Province, Nanchang, China \\ SHANSHAN WU \\ Jiangxi Climate Center, Nanchang, China \\ JIUSHENG SHAN \\ Jiangxi Meteorological Observatory, Nanchang, China \\ XUETING Yi \\ Meteorological Disaster Emergency Warning Center of Jiangxi Province, Nanchang, China
}

(Manuscript received 1 November 2018, in final form 14 June 2019)

\begin{abstract}
A method named BTREC, which is in fact an extension of tracking a radar echo by cross-correlation (TREC) method, is proposed based on the Barnes filter in this paper. BTREC is an efficient objective analysis method for smoothing the motion vectors of radar echo patterns. A comparative analysis of the BTREC vectors and the COTREC (another extension of TREC, which is restricted by the 2D continuity equation) vectors is conducted. The results show that the BTREC method corrects the noise and inconsistencies in TREC vectors (often induced by shielding, ground clutter, and rapid morphological changes of the radar patterns) more thoroughly than COTREC. Then the BTREC, COTREC, and TREC methods are applied to extrapolate the real radar echo pattern over Jiangxi Province in China for two cases (1900-2100 UTC 4 June and 0900-1100 UTC 30 June 2018). Results show that the BTREC method performs the best out of the three methods to forecast the radar echo patterns in the following 1 and $2 \mathrm{~h}$, and has the least distortion. To further confirm that, 892 radar composite reflectivity mosaic images with precipitation in summer (June-August) over Jiangxi are collected to test the three methods. The results show that in the 1- and 2-h extrapolations, the mean differences of the threat score (TS), correlation (CORR), and probability of detection (POD) between BTREC and TREC are obviously more than 0, while that of the false alarm ratio (FAR) is remarkably less than 0 (the threshold to identify whether a grid is correctly predicted is set to $10 \mathrm{dBZ}$ ). Although the mean difference of TS, CORR, POD, and FAR between COTREC and TREC have similar variation, their magnitudes are obviously decreased (especially for the 2-h extrapolation). This further indicates that the BTREC method obviously improves the forecast of the radar echo pattern within the next $2 \mathrm{~h}$ compared to the COTREC and TREC methods.
\end{abstract}

\section{Introduction}

The description of the atmospheric-state evolution is an inherent three-dimensional problem involving several variables such as temperature, humidity, water vapor, and rainwater (Bechini and Chandrasekar 2017). Therefore, the nowcasting of precipitation and wind can be performed by the use of numerical weather prediction (NWP) models. However, the NWP-based

Corresponding author: Haibo Zou, zouhaibobo@sohu.com nowcasting presents low skills because of the difficulties in depicting small-scale convective features in the model initial conditions (i.e., the model spinup problem; Hwang et al. 2015). In addition to NWP, nowcasting can also be accomplished by radar echo extrapolation which is in fact based on the inertia motion of the radar echo pattern. In contrast, such extrapolation-based nowcasting techniques, which are in fact two-dimensional forecasts of rainwater, are more skillful than NWP-based (Lin et al. 2005; Mandapaka et al. 2012). Although data (such as radar and satellite) assimilation could improve 
the skill of NWP models for short-range forecasts (Ballard et al. 2016), the NWP-based nowcasting still shows poor performance compared to the extrapolationbased nowcasting (Germann and Zawadzki 2002; Hwang et al. 2015). To attempt to improve the skill of standard extrapolation-based nowcasting, the blending techniques using extrapolation-based and NWP-based nowcasting have been developed (Liang et al. 2010; Seed et al. 2013; Hwang et al. 2015). However, the blending method does not improve the $0-1$-h forecast skill due to the poor performance of the NWP-based method (Liang et al. 2010).

Extrapolation-based nowcasting can be divided into two major sorts (Liang et al. 2010). The first sort is tracking the centroids of thunderstorm cells or the borders of intense echoes, such as TITAN (Thunderstorm Identification, Tracking, Analysis and Nowcasting; Dixon and Wiener 1993) and SCIT (Storm Cell Identification and Tracking; Johnson et al. 1998). The other sort focuses on the motion vectors of echo pattern, which are derived by tracking the radar echo by cross-correlation (TREC) technique (Rinehart and Garvey 1978) or optical flow technique (Bechini and Chandrasekar 2017; Woo and Wong 2017). The second sort extrapolates the whole echo field instead of the border or centroid of a thunderstorm cell. Therefore, they (especially for TREC) are widely applied in extrapolationbased nowcasting, and are equipped into many nowcasting systems such as the ITWS (Integrated Terminal Weather System; Evans and Ducot 1994), NIMROD (Nowcasting and Initialization for Modeling Using Regional Observation Data System; Golding 1998), NCAR ANC (Auto-Nowcaster; Mueller et al. 2003), SWIRLS (Short-range Warning of Intense Rainstorms in Localized Systems; Wong 2006), and BJ-ANC (Beijing-Auto-Nowcasting; Chen et al. 2010).

However, noisy vectors caused by the shielding, ground clutters, and small-scale changes of radar echoes often occur in the TREC vectors (Rinehart 1981). These noisy vectors are accompanied by obvious divergent features, often inducing the distortion of extrapolated radar echo with the leading time increasing (Chen et al. 2009). Although the motion vectors are not necessarily nondivergent around convective cells, removing the divergent component of motion vectors can improve the forecast performance, because it can smooth erroneous motion vectors and small-scale divergent features and improve the consistency of the resulting vector field ( $\mathrm{Li}$ et al. 1995). The small-scale divergent features, which may be induced by the real change of radar patterns, are not predictable on time scales of interest (Otsuka et al. 2016). Therefore, it is necessary to eliminate noisy vectors in TREC vectors. To smooth out them, Li et al. (1995) developed the continuity of TREC vectors (COTREC) technique using a variational method that constrains the TREC vectors by the 2D Boussinesq mass continuity equation. The COTREC technique improves the performance of extrapolated radar echo (Li et al. 1995; Chen et al. 2009) and then is widely applied to operational nowcasting systems such as CHMI (Czech Hydrometeorological Institute) nowcasting (Kyznarová and Novák 2009) and SWAN (Severe Weather Automatic Nowcast System; $\mathrm{Hu}$ et al. 2012). Although the COTREC method can significantly reduce the noise in TREC vectors, it cannot entirely remove the influence of zero vectors, small vectors, and vectors opposite to the surrounding vectors (Li et al. 1995). Huang et al. (2017) also indicated that the iteration of COTREC (solving a Poisson equation) can appear nonconvergent in typhoon weather due to the strong wind speed. Moreover, the iterative calculation of COTREC also requires more computation time.

In addition to the variational method based on the constraint of the 2D Boussinesq mass continuity equation, spatial filters can also smooth out noise in a $2 \mathrm{D}$ variable field. For example, Zhao et al. (2007) smoothed the noise in the radar echo images using the median filter, which replaced each pixel value with the median value of the local neighborhood pixels. Prior to performing COTREC algorithm, Otsuka et al. (2016) used Lanczos filter (a differential filter based on the Fourier transform) to smooth out random noise in high-resolution TREC vectors originated from phased-array weather radar observations. Chen et al. (2017) employed the bilateral filter to perform the quality control of radar echo noise. The Barnes filter, which was developed by Barnes (1964) based on Gaussian weighting function and improved by Barnes (1973), can separate signals with different scale from a 2D variable field. Based on it, Zhang et al. (2014) successfully extracted mesoscale vortices in southwestern China, and Zou et al. (2018a) commendably removed the small-scale perturbations in the output of numerical simulation, improving the positioned accuracy of the typhoon center after landfall. Among these filters, the performance of the median filter is not better than that of the Gaussian filter (Chen et al. 2017). The Lanczos filter is based on the Fourier transform and requires good continuity of data (Duchon 1979). The bilateral filter is developed on the basis of the Gaussian filter by introducing the luminance difference for retaining the information at the strong echo edges (Overton and Weymouth 1979; Chen et al. 2017). Moreover, the response function of bilateral filter is complex and depends on not only the spatial information but also the luminance difference. 
The improved Barnes filter (hereafter, Barnes filter) can suppress the small-scale noise, achieve the desired scale resolution with only one iteration (which can significantly reduce the computational time), and easily compute the response function (Maddox 1980). Askelson et al. (2000) indicated that the Barnes filter may be preferable for processing radar data over the Gressman filter due to the easy computation of the response function.

In view of the advantages of the Barnes filter, this work intends to eliminate noise in TREC vectors by using the Barnes filter (hereafter, BTREC) rather than a constraint of the 2D Boussinesq mass continuity equation, and hopes to further improve the performance of radar echo extrapolation. The remainder of this paper is organized as follows. Section 2 describes the data and processing. Section 3 reviews the TREC and COTREC techniques. Section 4 illustrates the BTREC algorithm. The results are given in section 5. Conclusions are given in section 6 .

\section{Data and processing}

China's Jiangxi Province and its surrounding areas (Fig. 1), situated in the East Asian monsoon region, are frequently affected by short, intense precipitation; tropical cyclones; strong winds; and hail in spring and summer. Radar data in this region are used to derive the BTREC vectors and test its extrapolated performance. The region includes 17 China New Generation Weather Radars (CINRADs) with $\mathrm{S}$ band of type A (i.e., CINRAD/SA, which is similar to the WSR-88D) and 2 CINRADs with $\mathrm{S}$ band of type $\mathrm{C}$ (i.e., CINRAD/SC, which is produced by 784 Factory in Chengdu, China; Fig. 1). The volume scan data (level II) with the temporal resolution of $6 \mathrm{~min}$ measured by the 19 CINRADs are obtained from the Meteorological Information Center of Jiangxi Province, China. These volume scan data are measured by the volume coverage pattern (VCP) 21 , including nine elevation angles $\left(0.5^{\circ}, 1.5^{\circ}, 2.4^{\circ}, 3.4^{\circ}, 4.3^{\circ}, 6.0^{\circ}, 9.9^{\circ}, 14.6^{\circ}\right.$, and $\left.19.5^{\circ}\right)$.

Owing to the significant influence of nonmeteorological echoes (isolated echoes and ground clutter) on TREC vectors (Rinehart 1981), they need to be removed from the raw radar data by the improved quality control method of radar reflectivity (Zou et al. 2018b). After removing nonmeteorological echoes, the radar data (level II) in polar coordinates (1-km resolution in range and about $1^{\circ}$ resolution in azimuth) are converted into a Cartesian coordinate with a horizontal resolution of $0.01^{\circ} \times 0.01^{\circ}$ based on the nearest-neighbor interpolation method on the range-azimuth plane (Zou et al. 2014). In practice, the composite reflectivity (the maximum

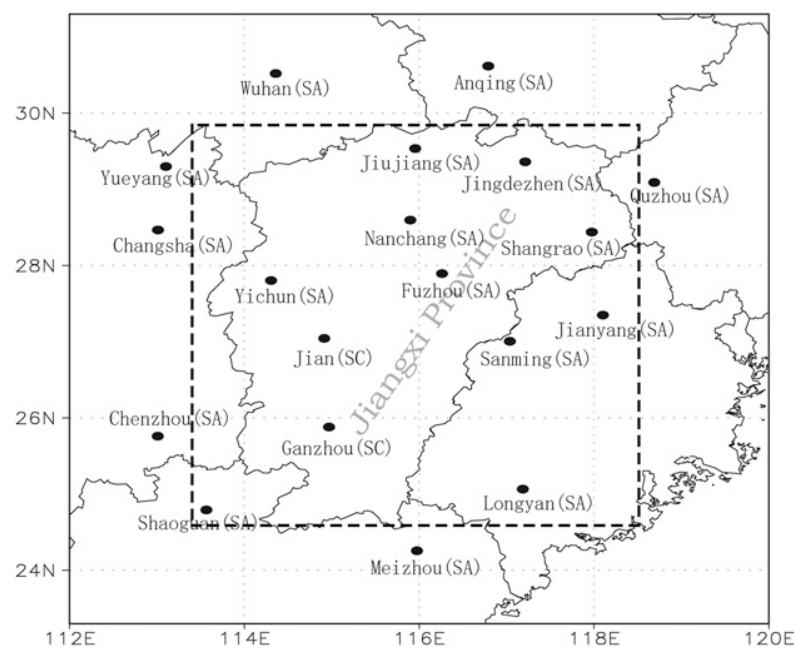

FIG. 1. Map of CINRAD stations (filled circles) in Jiangxi Province (China) and its surrounding areas. SA (SC) indicates that the radar format is $\mathrm{S}$ band of type A (type $\mathrm{C}$ ). The dashed rectangle represents the area in which the observations and forecasts are used to calculate the verification indices.

reflectivity from all elevation angles of the radar data) is usually exploited to monitor the convective weather because the radar beams at low elevation angle are often blocked by mountains near the radar. As a result, the mosaic of composite reflectivity of the 19 radars is chosen to analyze BTREC and its performance. The horizontal resolution of the mosaic is $0.01^{\circ} \times 0.01^{\circ}$. The coverage area is $23.3-31.3^{\circ} \mathrm{N}, 112^{\circ}-120^{\circ} \mathrm{E}$ (the corresponding grid resolution is $801 \times 801$ in zonal and meridional directions), as shown in Fig. 1. In addition, the mosaic is accomplished by using the maximum value of the composite reflectivity observed by all the radars (Wu et al. 2018).

\section{Review of TREC and COTREC}

\section{a. TREC}

The TREC method was developed by Rinehart and Garvey (1978). The method computes the movement vectors (TREC vectors) of radar echo patterns within a short time interval $\Delta t$ and thus it requires two composite reflectivity fields with an interval of several minutes. To calculate the TREC vectors, the composite reflectivity field at the first time $t_{1}$ is divided into a number of equally sized boxes by a given distance. Each box at $t_{1}$ (such as the solid rectangle in Fig. 2a) is then compared with all the possible boxes of the same size at $t_{2}$. This comparison is based on the correlation coefficient between the reflectivity values in the boxes at two times. The box with the highest correlation coefficient at $t_{2}$ (i.e., the solid rectangle in Fig. $2 \mathrm{~b}$ ) is 


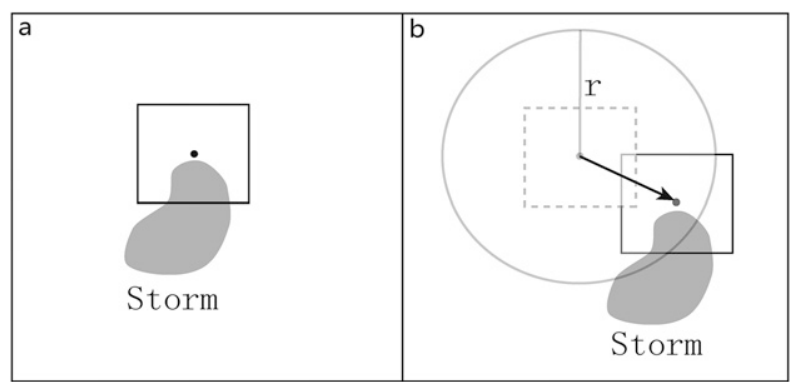

FIG. 2. Schematic map of the TREC method at (a) time $t_{1}$ and (b) time $t_{2}$. The box at $t_{1}$ [i.e., the solid box in (a) or dotted box in (b)] is compared to all the boxes within a searching-distance $r$ at $t_{2}$. The position of the matching box with the largest correlation coefficient is the endpoint of the TREC vector [i.e., arrow in (b)]

selected as the destination of the given box at $t_{1}$ (i.e., the initial box) during the period of $\Delta t=t_{2}-t_{1}$. The vector from the center of the initial box to that of the destination box (i.e., the vector in Fig. 2b) is defined as the TREC vector, which indicates the motion vector of the storm in the initial box during the period of $\Delta t$. Since the motion of a storm has a certain velocity, the procedure to search the best-matching box at $t_{2}$ is not necessarily performed in the entire dataset. The search area should be limited to the circle region with the center at the initial box center (i.e., the circle in Fig. 2b) and the radius of $r$. The value of $r$ is determined by the likely maximum velocity $V_{\max }$ of a storm and the time interval $\Delta t$. In general, $V_{\max }$ is less than $30 \mathrm{~m} \mathrm{~s}^{-1}$ (Chen et al. 2009). It is noteworthy that the spatial resolution and the time interval $\Delta t$ of the composite reflectivity are $0.01^{\circ} \times 0.01^{\circ}$ and $12 \mathrm{~min}$, respectively. The matching box size is set as $0.15^{\circ} \times 0.15^{\circ}$ (about $15 \mathrm{~km} \times 15 \mathrm{~km}$ ) which is the recommended size for obtaining optimal results (Rinehart 1981). Although tuning the matching box size may further improve the quality of TREC vectors in this application, it is beyond the scope of this paper. Besides, in order to save computing time, the TREC vectors are computed at every $15 \times 15$ grid points without overlapping the matching box.

The TREC vectors often show an apparently inconsistent and noisy behavior, which is mainly induced by ground clutter, shielding, and failures in tracking radar echo patterns. The quality control of the radar reflectivity to remove ground clutter only solves the problem partially $(\mathrm{Li}$ et al. 1995). To reduce the apparently incorrect vectors, a TREC vector that deviates more than $25^{\circ}$ from the mean direction of the neighboring vectors (eight points) is replaced by the average of the neighboring vectors ( $\mathrm{Li}$ et al. 1995). This is also done for a vector with zero velocity.

\section{b. COTREC}

Li et al. (1995) used a variational technique that forced 2D continuity equation on TREC vectors to correct noisy vectors and improve the consistency of the resulting vector field. This method is well known as COTREC. The variational technique was first applied by Sasaki $(1958,1970)$ in meteorology. A number of variational techniques have since been developed and widely applied to the retrieval of meteorological fields (Ray 1980) and assimilation systems (Barker et al. 2004). It can make the outcome close to the observations at the locations where observations are available, and meanwhile the outcome can fulfill the imposed dynamical or statistical constraints. The COTREC method corrects noise and inconsistencies in TREC vectors, reducing the divergence of the resulting vector field ( $\mathrm{Li}$ et al. 1995). Specifically, the classical solution of a variational analysis can be expressed in terms of minimizing a cost function $J$ :

$$
J=V(u, v)=\int\left[\left(u-u^{0}\right)^{2}+\left(v-v^{0}\right)^{2}\right] d x d y .
$$

The constraint of two-dimensional continuity equation is considered as

$$
\frac{\partial u}{\partial x}+\frac{\partial v}{\partial y}=0
$$

The variables $u^{0}$ and $v^{0}$ are the zonal and meridional components of the TREC vectors, respectively, and $u$ and $v$ are the solution of the variational analysis (i.e., the COTREC vector). The problem to solve Eq. (1) (i.e., determining the minimum $J$ ) under the constraint of Eq. (2) is equivalent to the problem to find the stationary points of the following scalar function with respect to the three variables $u, v$, and $\lambda$ (Bertsekas 1982; $\mathrm{Li}$ et al. 1995):

$$
\begin{aligned}
F(u, v, \lambda)= & \int\left[\left(u-u^{0}\right)^{2}+\left(v-v^{0}\right)^{2}\right. \\
& \left.+\lambda\left(\frac{\partial u}{\partial x}+\frac{\partial v}{\partial x}\right)\right] d x d y
\end{aligned}
$$

where $\lambda$ is usually denoted as the Lagrangian multiplier. Thus, the minimization of the cost function $J$ under the constraint of Eq. (2) is converted into an unconstrained minimization of $F$.

The associated solution for minimizing $F$ is as follows:

$$
\begin{aligned}
\frac{\partial^{2} \lambda}{\partial^{2} x}+\frac{\partial^{2} \lambda}{\partial^{2} y} & =-2\left(\frac{\partial u^{0}}{\partial x}+\frac{\partial v^{0}}{\partial y}\right), \\
u & =u^{0}+\frac{1}{2} \frac{\partial \lambda}{\partial x} \\
v & =v^{0}+\frac{1}{2} \frac{\partial \lambda}{\partial y}
\end{aligned}
$$


Equation (4) is a partial differential equation of elliptic type (more specifically, it is the Poisson equation), which can be solved by the successive overrelaxation (SOR) method (Press et al. 1991). The SOR method can give a fast convergence to the solution. The boundary condition of $\lambda$ is set to 0 , and the adjusted motion (i.e., the COTREC) vectors are then obtained by using the following difference formulation of Eqs. (5) and (6) ( $\mathrm{Li}$ et al. 1995):

$$
\begin{aligned}
& u_{i, j}=\frac{1}{4}\left(u_{i+1, j}^{0}+2 u_{i, j}^{0}+u_{i-1, j}^{0}\right)+\frac{1}{2}\left(\frac{\lambda_{i+1, j}-\lambda_{i-1, j}}{2 \Delta x}\right), \\
& v_{i, j}=\frac{1}{4}\left(v_{i, j+1}^{0}+2 v_{i, j}^{0}+v_{i, j-1}^{0}\right)+\frac{1}{2}\left(\frac{\lambda_{i, j+1}-\lambda_{i, j-1}}{2 \Delta y}\right) .
\end{aligned}
$$

\section{BTREC}

The presented BTREC method is designed to correct noisy and inconsistent TREC vectors. It is in fact the use of the Barnes filter on TREC vectors to remove smallscale signals (noise) and conserve the movement signals of storms. The process of BTREC includes two steps. The first step is the selection of parameters in the Barnes filter so that it can remove noisy and inconsistent signals in TREC vectors but preserve the movement signals of a storm. The second step is the removal of small-scale noisy TREC vectors based on a Barnes filter whose parameters have been appropriately selected.

\section{a. Introduction to the Barnes filter}

Assuming that the distribution of an atmospheric variable can be described by a Fourier integral, Barnes (1964) proposed a filter based on a Gaussian weighting function to separate different-scale signals from a $2 \mathrm{D}$ variable. Barnes (1973) then improved this filter and required just one iteration rather than several successive iterations, significantly reducing the computational time. A number of studies have shown its excellent performance in separating signals with different scales from a 2D meteorological field (Gomis and Alonso 1990; Morris and Smith 2001; Zhang et al. 2014). Based on the Barnes filter, Zou et al. (2018b) removes the small-scale signals with wavelengths less than $100 \mathrm{~km}$ in the numerical model output, improving the position accuracy of the TC center after landfall. The expressions of the modified Barnes filter (Barnes 1973; Doswell 1977) are as follows:

$$
F_{0}(x, y)=\frac{\sum_{k=1}^{M} w_{k} F\left(x_{k}, y_{k}\right)}{\sum_{k=1}^{M} w_{k}},
$$

$$
\begin{aligned}
w_{k} & =\exp \left(-\frac{r_{k}^{2}}{4 C}\right) \\
F_{1}(x, y) & =F_{0}(x, y)+\frac{\sum_{k=1}^{M} w_{k}^{\prime}\left[F\left(x_{k}, y_{k}\right)-F_{0}\left(x_{k}, y_{k}\right)\right]}{\sum_{k=1}^{M} w_{k}^{\prime}} \\
w_{k}^{\prime} & =\exp \left(-\frac{r_{k}^{2}}{4 G C}\right)
\end{aligned}
$$

where $F(x, y)$ is the zonal $(x)$ or meridional (y) component of TREC vectors (i.e., $u^{0}$ or $\left.v^{0}\right) ; F_{0}(x, y)$ is the initial filter value of $F(x, y)$; and $F_{1}(x, y)$ is the final filter value of $F(x, y)$ (i.e., BTREC vectors). Variable $M$ is the grid number with valid TREC vector within an influence range (default: $100 \mathrm{~km}$ ) of the grid point $(x, y), w_{k}$ is the Gaussian weight function, and $w_{k}^{\prime}$ is the modified Gaussian weight function. Variable $r_{k}$ is the distance between point $(x, y)$ and point $\left(x_{k}, y_{k}\right)$, and $G$ and $C$ are the filter parameters. The influence of data may be extended to any distance without changing the weight function and response characteristics, because the Gaussian weight function $w_{k}$ [i.e., Eq. (2)] asymptotically approaches zero (Maddox 1980).

\section{b. The selection of filter parameters}

To extract a desirable signal from a raw 2D field, the appropriate parameters $G$ and $C$ should be chosen before the Barnes filter is performed because they can influence the response of the Barnes filter. In fact, the response is the function of $G, C$, and wavelength $\lambda$ and can be expressed as follows (Doswell 1977; Maddox 1980):

$$
R=R_{0}\left(1+R_{0}^{G-1}-R_{0}^{G}\right)
$$

and

$$
R_{0}=\exp \left(-\frac{4 \pi^{2} C}{\lambda^{2}}\right)
$$

The larger the response of $R$, the more retainment of the signals at the wavelength of $\lambda$, and vice versa. Generally, $R>0.5(<0.5)$ indicates the signals of corresponding wavelength $\lambda$ are basically conserved (removed) in the field (Gomis and Alonso 1990; Zhang et al. 2014). Equations (13) and (14) imply that the responses of the Barnes filter are only determined by the parameters $C$ and $G$ in a given wavelength of $\lambda$, and $G$ is between 0 and 1 (Barnes 1964; Maddox 1980). Moreover, the response is difficult to converge fast 

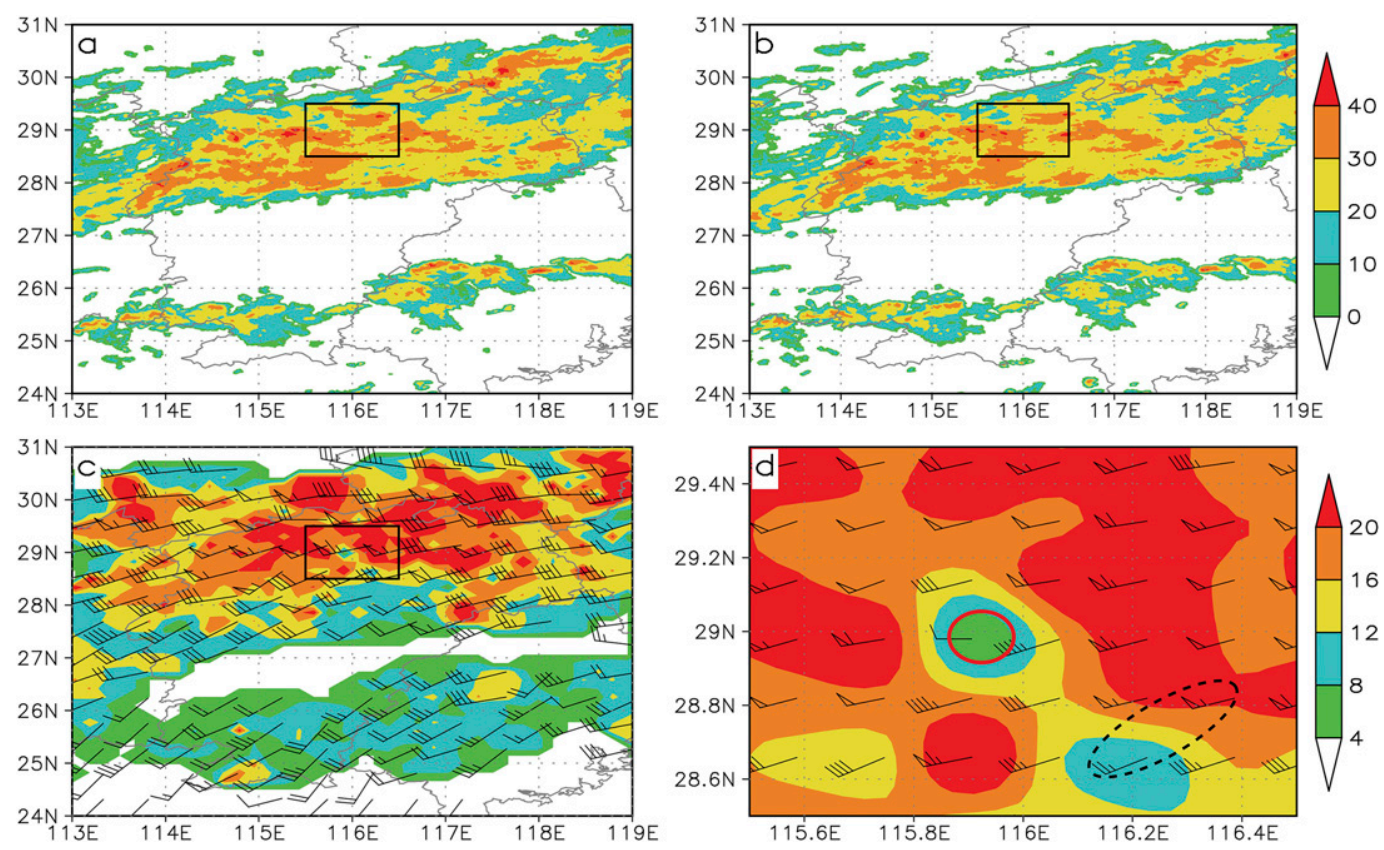

FIG. 3. Composite reflectivity (dBZ) at (a) 1848 and (b) 1900 UTC 4 June 2018, (c) the retrieved TREC vectors and the zonal component $\left(\mathrm{m} \mathrm{s}^{-1}\right)$, and (d) the enlarged view of black rectangle region in (c). Full and half vector barbs denote 4 and $2 \mathrm{~m} \mathrm{~s}^{-1}$, respectively.

when $G>0.5$. Actually, $G=0.35$ is widely used in practice (Maddox 1980; Gomis and Alonso 1990; Zhang et al. 2014; Zou et al. 2018a). Hence, $G=0.35$ is also applied in this study. The response function only depends on the parameter $C$.

There is always noise in the TREC vectors due to the shielding, ground clutter, and small-scale changes of the radar echo patterns (Rinehart 1981). Figures 3a and $3 \mathrm{~b}$ show the radar echo pattern at 1848 and 1900 UTC 4 June 2018. Figure $3 \mathrm{c}$ depicts the TREC vectors and its zonal components obtained from the two radar echo patterns. It is noted that the horizontal resolution of radar data is about $1 \mathrm{~km}$, and the box size and search radius of TREC pattern matching is 15 and $21 \mathrm{~km}$, respectively. It is clear that the motion vectors over Jiangxi Province of China are westerly vectors or southwesterly vectors with the speed ranging from 4 to $24 \mathrm{~m} \mathrm{~s}^{-1}$ (Fig. 3c). These vectors preferably reflect the overall movement of radar echoes over Jiangxi. However, there are obvious convergences or divergences in these vectors, such as the areas in the black rectangle in Fig. 3c. To display the TREC vectors more clearly, the black rectangle region in Fig. $3 \mathrm{c}$ is enlarged into Fig. 3d. In the red circle in Fig. 3d, the TREC vector is about $4 \mathrm{~m} \mathrm{~s}^{-1}$, but the adjacent TREC vectors are more than $16 \mathrm{~m} \mathrm{~s}^{-1}$, even as high as $24 \mathrm{~m} \mathrm{~s}^{-1}$. This TREC vector is a noise likely induced by the failure in tracking radar echo patterns due to the small-scale changes of radar echo patterns. The noisy vector in the red circle can generate significant divergence and convergence in the neighboring area. The velocity difference in the zonal component of TREC vectors between two adjacent grids (near the red circle) can reach to $20 \mathrm{~m} \mathrm{~s}^{-1}$ (Fig. 3d). Moreover, in the neighboring regions of the red circle the flows can be characterized by the obvious horizontal shear of TREC vectors (Fig. 3d).

Figures $3 \mathrm{c}$ and $3 \mathrm{~d}$ show that the noisy TREC vectors (i.e., the isolated abnormally large or small velocity center) mainly consist of one point (at most two points) in a local region, indicating that the horizontal scale of noisy TREC vectors is less than $50 \mathrm{~km}$ and that of the movement of radar echo pattern is more than $50 \mathrm{~km}$ or even more than $100 \mathrm{~km}$. Consequently, the signals with horizontal scale less than $50 \mathrm{~km}$ should be removed while the signals with horizontal scale more than $50 \mathrm{~km}$ (especially more than $100 \mathrm{~km}$ ) should be preserved. What is the value of parameter $C$ that can make the Barnes filter doing so? Figure 4 shows the evolution of the response function of Barnes filter with different values of $C$. It is clear that the response increases with the wavelength in a given $C$, and in a fixed wavelength the response decreases with the increase of $C$. Specifically, the response of the Barnes filter at a wavelength of $50 \mathrm{~km}$ is less than 0.3 when $C \geq 300$. Meanwhile, the response at a wavelength of $100 \mathrm{~km}$ is more than 0.7 when $C \leq 300$. Since the continuous variation of the 


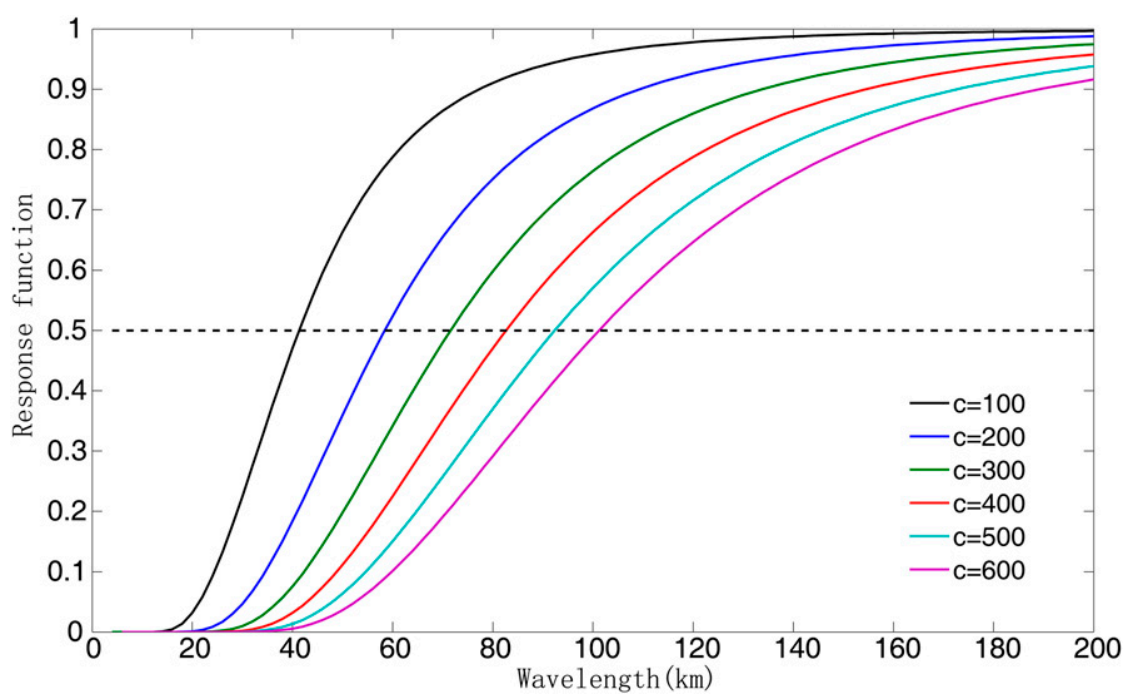

FIG. 4. Response function of the Barnes filter for parameter $C$ at $G=0.35$. The dashed line indicates a response function value of 0.5 .

response function with $C$ and wavelength, there is no value of $C$ that simultaneously meets the requirement that the response function is obviously less than 0.5 at wavelengths $<50 \mathrm{~km}$ and remarkably more than 0.5 at wavelengths $>50 \mathrm{~km}$. Therefore, selecting $C$ as 300 is very appropriate for the Barnes filter because it can remarkably remove the signals with a wavelength less than $50 \mathrm{~km}$ (the response is less than 0.2 ) and preserve the signals with a wavelength more than $80 \mathrm{~km}$ (the response is more than 0.6) in TREC vectors (Fig. 4). In contrast, the Barnes filter with $C=200$ cannot cleanly remove the signals with a wavelength less than $50 \mathrm{~km}$ because the response is about 0.5 , and the Barnes filter with $C=400$ cannot commendably preserve the signals with a wavelength more than $80 \mathrm{~km}$ because the response at wavelength of $80 \mathrm{~km}$ is less than 0.5 .

\section{Results}

\section{a. Motion vectors}

After removing noise (i.e., small-scale perturbations) by the Barnes filter, the improved TREC vectors (i.e., BTREC vectors) are smoother (Fig. 5a). Specifically, the velocity in the red circle significantly increased from about $4 \mathrm{~m} \mathrm{~s}^{-1}$ (Fig. 3d) to about $16 \mathrm{~m} \mathrm{~s}^{-1}$ (Fig. 5a), and meanwhile the velocity to the west of the red circle decreased from about 24 to about $18 \mathrm{~m} \mathrm{~s}^{-1}$. The difference of the zonal component of the BTREC vectors between any two adjacent grids is less than $3 \mathrm{~m} \mathrm{~s}^{-1}$ (Fig. 5a). Moreover, the comparison between Fig. $3 \mathrm{~d}$ and Fig. 5a shows that the Barnes filter can also reduce the horizontal shear of the TREC vectors. These indicate that the Barnes filter could smooth the inconsistences and noise in the TREC vectors.
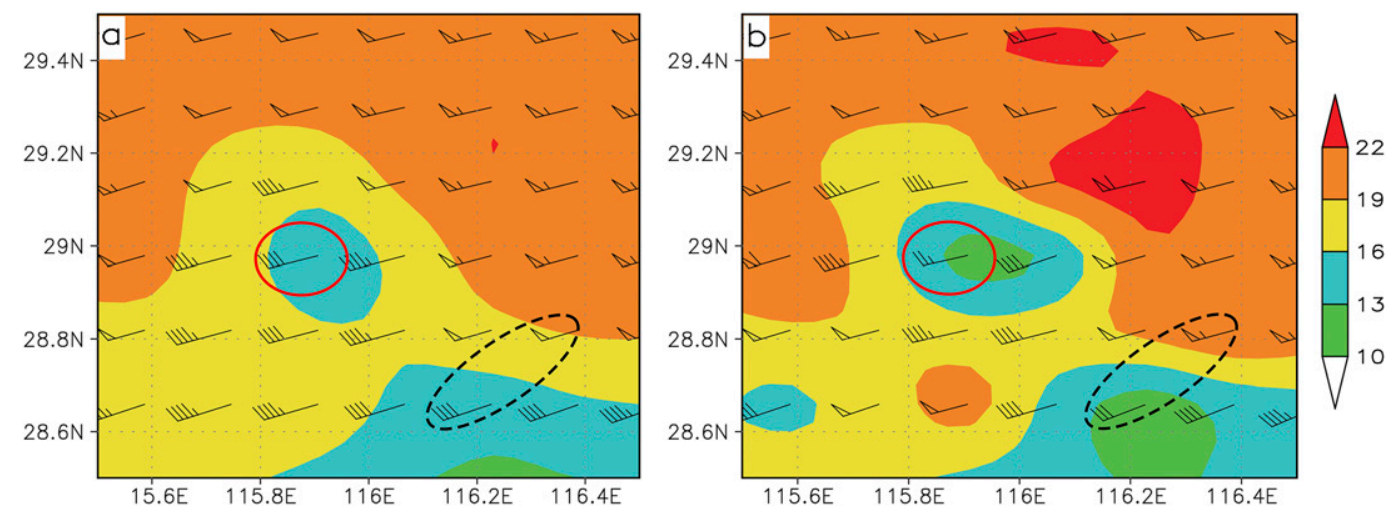

FIG. 5. (a) BTREC vectors and the zonal component (shaded; $\mathrm{m} \mathrm{s}^{-1}$ ) and (b) the COTREC vectors and the zonal component (shaded; $\mathrm{m} \mathrm{s}^{-1}$ ). Full and half vector barbs denote 4 and $2 \mathrm{~m} \mathrm{~s}^{-1}$, respectively. 

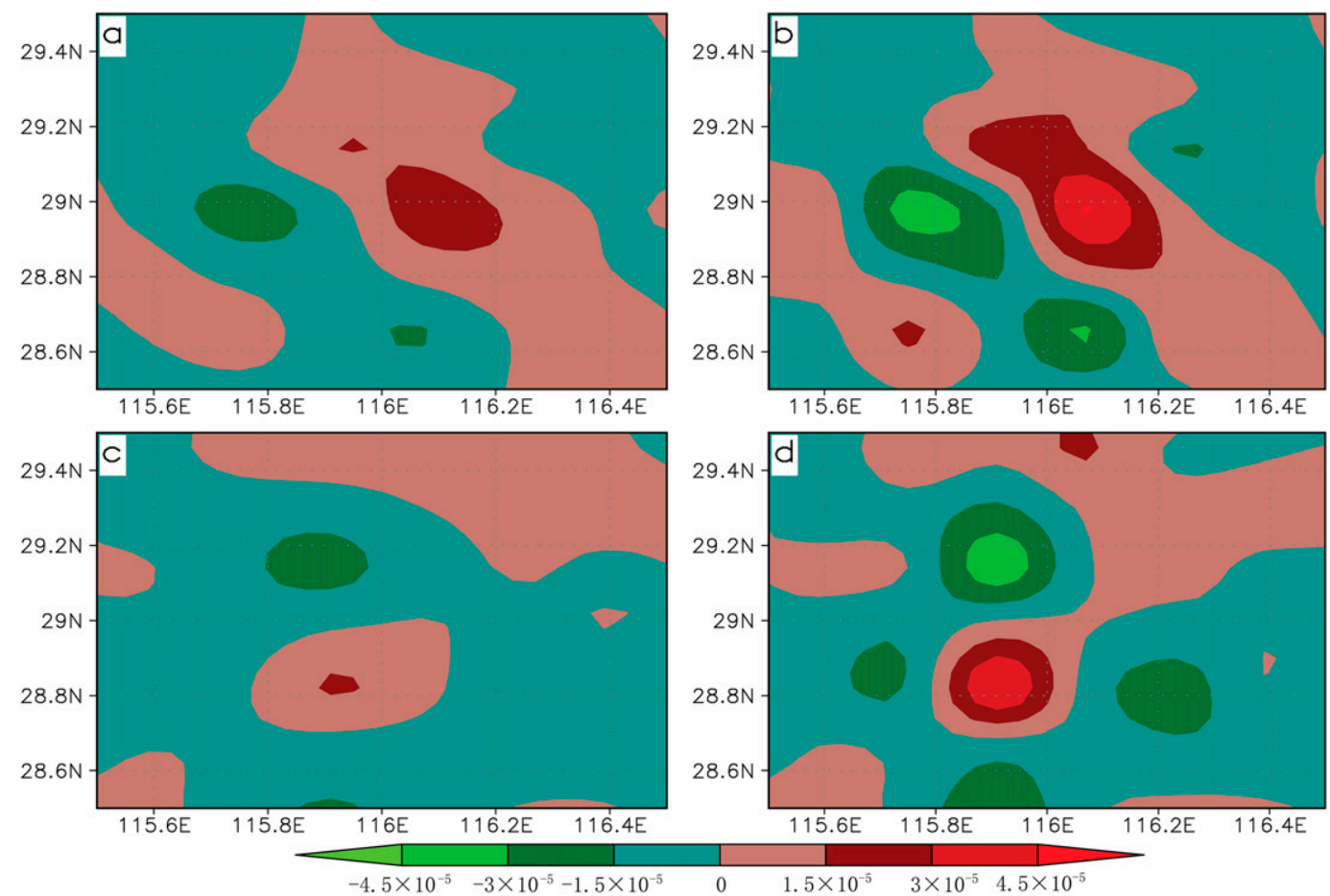

FIG. 6. (top) Divergence $\left(\mathrm{s}^{-1}\right)$ of (a) BTREC vectors and (b) COTREC vectors and (bottom) vorticities $\left(\mathrm{s}^{-1}\right)$ of (c) BTREC vectors and (d) COTREC vectors.

The COTREC vectors and its zonal components are presented in Fig. 5b. The COTREC method has also smoothed the TREC vectors, increasing (decreasing) the velocity of the vectors that are small (large) in Fig. 3d, reducing the horizontal shear of motion vectors (Fig. 5b). But, by comparison, the smoothed degree of the COTREC method is not as intense as that of the BTREC method. For example, BTREC increases the velocity of the noisy TREC vector in the red circle by about $12 \mathrm{~m} \mathrm{~s}^{-1}$ (from 4 to $16 \mathrm{~m} \mathrm{~s}^{-1}$ ) while COTREC increases it only by about $6 \mathrm{~m} \mathrm{~s}^{-1}$ (from 4 to $10 \mathrm{~m} \mathrm{~s}^{-1}$ ). A larger gradient of the zonal component of COTREC (Fig. 5b) than that of BTREC (Fig. 5a) further confirms this viewpoint. To quantitatively and comparatively analyze the stretching (or compressing) and rotating of the BTERC and COTREC vectors, the divergence and vorticity fields are calculated in the region of interest (Fig. 6). The divergences of BTREC vectors are between $-3 \times 10^{-5}$ and $3 \times 10^{-5} \mathrm{~s}^{-1}$ (Fig. 6a). It is obviously less than those of COTREC, which are between $-4.5 \times 10^{-5}$ and $6 \times 10^{-5} \mathrm{~s}^{-1}$ (Fig. 6b). Figures $6 \mathrm{c}$ and $6 \mathrm{~d}$ show that the vorticities of BTREC vectors (ranging from $-3 \times 10^{-5}$ to $3 \times 10^{-5} \mathrm{~s}^{-1}$ ) are also less than those of COTREC (ranging from $-4.5 \times$ $10^{-5}$ to $4.5 \times 10^{-5} \mathrm{~s}^{-1}$ ). The weak divergence and vorticity confirm that BTREC vectors are smoother than COTREC vectors, implying that the BTREC method can correct noise and inconsistencies in TREC vectors more thoroughly.

\section{b. Extrapolation}

The above analyses have shown that the BTREC method can correct noise and inconsistencies in TREC vectors more thoroughly than the COTREC method. This speculates that the extrapolated radar echo pattern based on BTREC vectors has fewer distortions than TREC vectors and COTREC vectors. But how does it work in practice? To address this problem, a case study and statistical analysis are implemented to assess the performance of extrapolation within $2 \mathrm{~h}$ in the following sections. Here, the motion vectors of the radar echo pattern (i.e., the TREC, COTREC, and BTREC vectors) are interpolated into the grids of radar composite reflectivity mosaic with a resolution of $0.01^{\circ} \times 0.01^{\circ}$, and then the backward scheme provided by Germann and Zawadzki (2002) is used to extrapolate the radar echo pattern in this paper.

\section{1) CAse study}

In this section two cases (the first one occurred at 1900-2100 UTC 4 June 2018, and the second occurred at 0900-1100 UTC 30 June 2018) over Jiangxi are used to test the performances of the BTREC, COTREC, and TREC methods. For the first case (i.e., the case of 

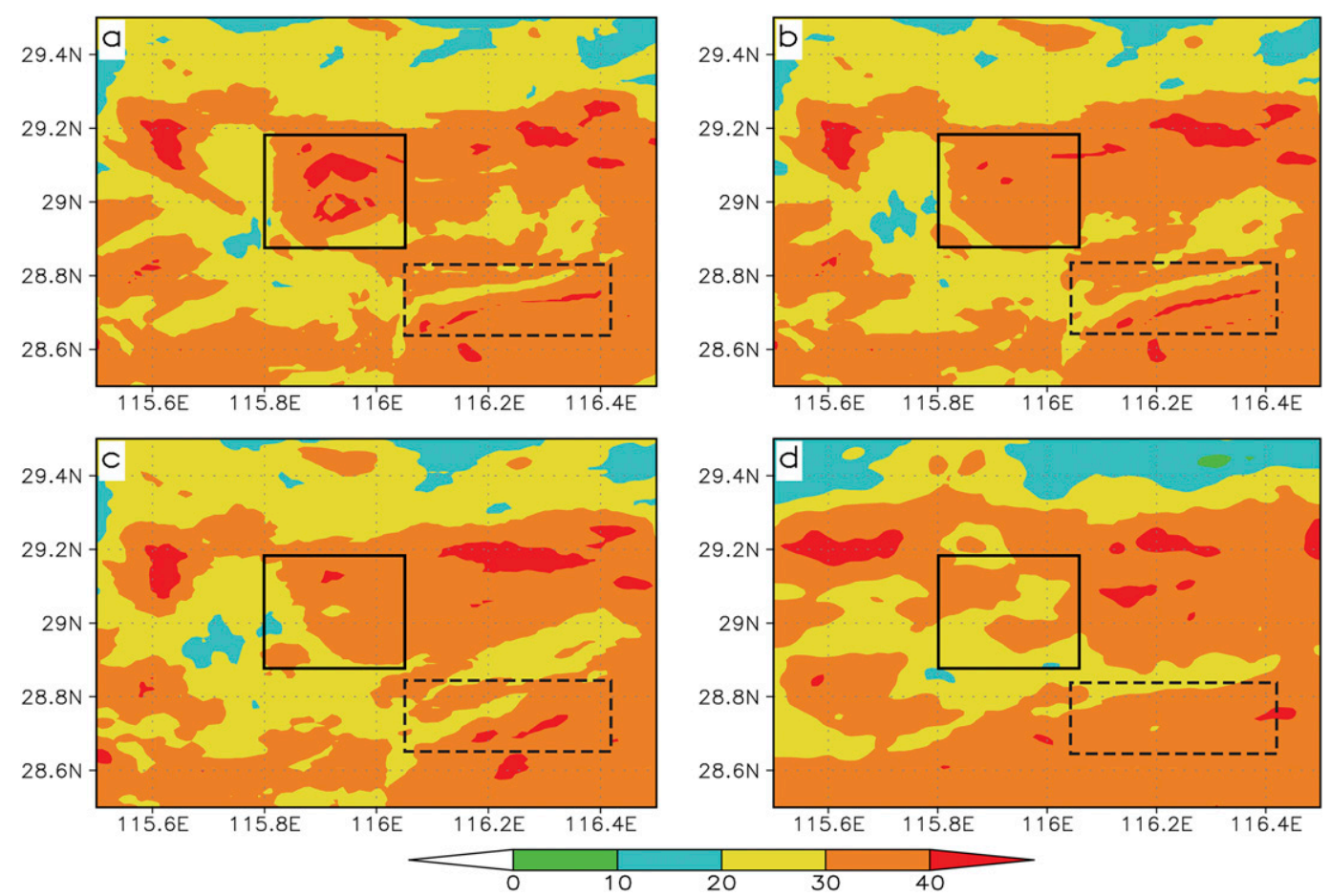

FIG. 7. Composite reflectivity (dBZ) of the 1-h extrapolations based on (a) TREC vectors, (b) COTREC vectors, and (c) BTREC vectors and (d) the observations at 2000 UTC 4 June 2018.

Figs. 3, 5, and 6), the 1-h extrapolations based on the three methods (TREC, COTREC, and BTREC) and observations at 2000 UTC 4 June 2018 are shown in Fig. 7. It is clear that all three methods successfully extrapolate the radar echo pattern. However, in the solid black rectangle region the TREC method extrapolated a ring with reflectivity more than $40 \mathrm{~dB} Z$ (Fig. 7a), but such a ring does not appear in the extrapolations of the COTREC (Fig. 7b) and BTREC (Fig. 7c) and the observation (Fig. 7d). Clearly, this ring is abnormal echoes and is induced by the extrapolated distortion of the TREC method. That the ring cannot be found in the extrapolations of the COTREC and BTREC (Figs. 7b,c) suggests that the COTREC and BTREC are better than the TREC to extrapolate the radar echo pattern. More importantly, there are two southwest-northeast-oriented linear echo belts (one's intensity is between 20 and $30 \mathrm{~dB} Z$, and the other one's intensity is more than $40 \mathrm{dBZ}$ ) in the dashed rectangle region in Figs. 7a and 7b. Such deformed linear echo belts are induced by the intense divergence of southwesterly flows in the black dashed ellipse area in Fig. 3d (TREC vectors) and Fig. 5b (COTREC vectors). By comparison, BTREC vectors (Fig. 5a) have a very small divergence of southwesterly vectors in the black dashed ellipse area and do not induce the abnormal linear echo belt in the dashed rectangle region in Fig. 7c.
This suggests that although the COTREC method eliminates the noise and inconsistencies in TREC vectors and improves the performance of extrapolation, the BTREC method does better.

When the extrapolation time (i.e., leading time) is prolonged to $2 \mathrm{~h}$, the distortions become more distinct in the extrapolations of TREC and COTREC. In the extrapolation of TREC, the abnormal circular echoes in the solid black rectangle in Fig. 7a develop into an "eye" shape, and the initiation of a new abnormal circular echo is apparent in the south to the "eye" (Fig. 8a). At the same time, an abnormal circular echo also appears in the extrapolation of the COTREC (i.e., the black rectangle in Fig. 8b, which is the "eye" area in Fig. 8a). The abnormal southwest-northeast-oriented linear echo belt with reflectivity more than $40 \mathrm{~dB} Z$, which located southeast of the black rectangle in Fig. 7b, is clearer (Fig. 8b). By comparison, there are no obvious abnormal echoes in the extrapolation of the BTREC method, especially for the area in the black rectangle and to its southeast (Fig. 8c). In fact, the abnormal circular echoes or linear echoes in Fig. 8a (Fig. 8b) are mainly induced by the large divergence and intense shear of TREC (COTREC) vectors near the red circle and black dashed ellipse in Fig. 3d (Fig. 5b). It is clear that the BTREC method can obtain more reasonable (few distortions) extrapolation 

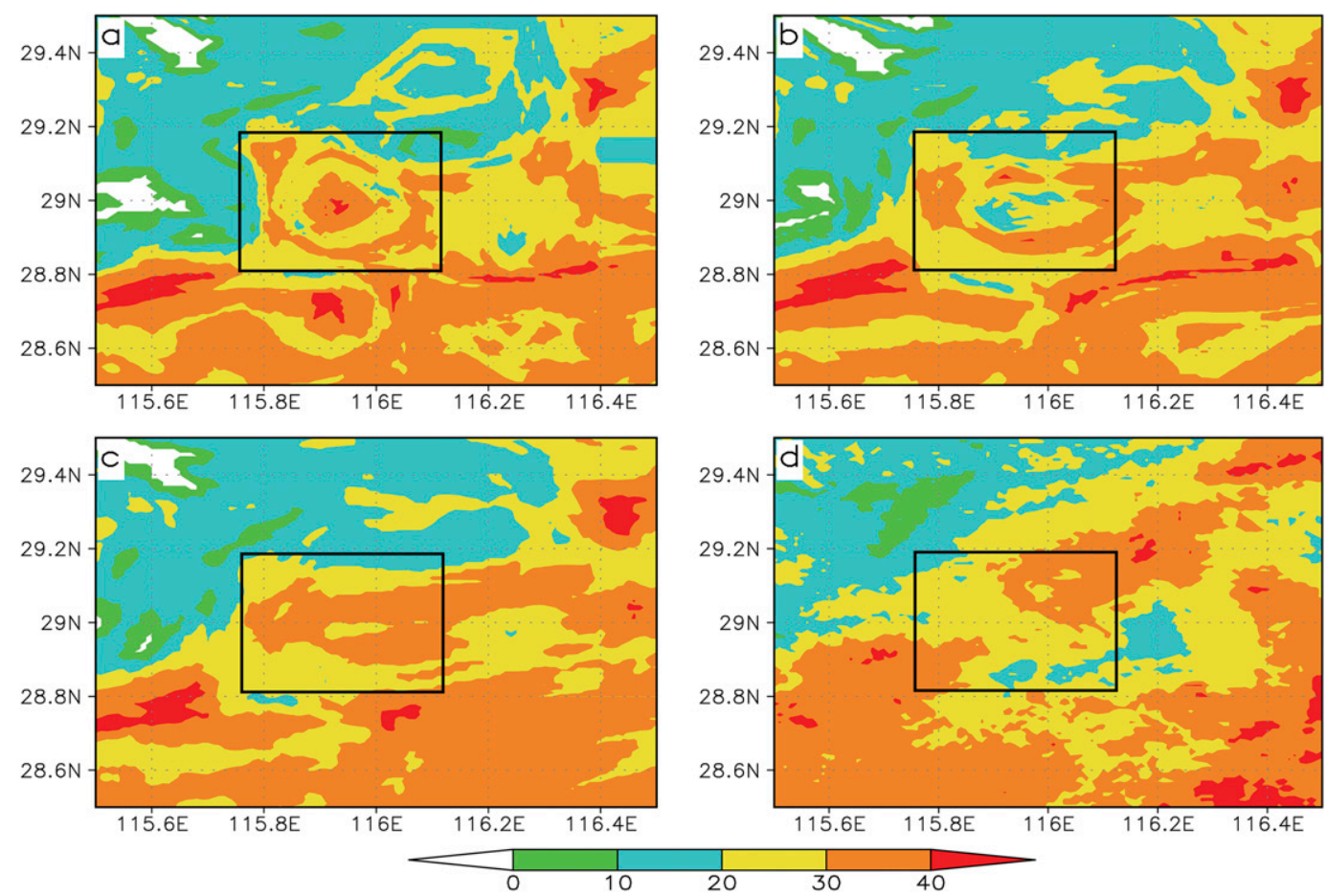

FIG. 8. Composite reflectivity (dBZ) of the 2-h extrapolations based on (a) TREC vectors, (b) COTREC vectors, and (c) BTREC vectors and (d) the observations at 2100 UTC 4 June 2018.

with 1- and 2-h lead time because it can smooth out the noise and inconsistencies more thoroughly than the COTREC method (Fig. 5).

Figure 9 shows the 2-h extrapolation of the other case. The observational echo pattern at 1100 UTC 30 June 2018 is shown in Fig. 9d, and the extrapolations with 2-h lead time based on TREC, COTREC, and BTREC are shown in Figs. 9a-c, respectively. Some abnormal circular echoes are presented distinctly in the extrapolation of TREC, such as the areas encircled by the dashed rectangles (Fig. 9a). Although the COTREC method obviously reduces the number of abnormal circular echoes, several initial abnormal circular echoes still appear in Fig. 9b, such as the areas encircled by the dashed rectangle. By contrast, few abnormal circular echoes occur in the extrapolation of BTREC (Fig. 9c). This case also suggests that the BTREC method performs the best of the three methods (fewest distortions) in extrapolating the radar echo pattern out to $2 \mathrm{~h}$.

\section{2) Statistical ANALySES}

The above case studies show that the BTREC method produces a more reasonable extrapolation of the radar echo pattern than the traditional COTREC and TREC methods. Is this improvement universal or just case dependent? To address this question, 892 mosaic images of radar composite reflectivity with precipitation (of about 2500 stations of Jiangxi Province, more than 10 stations have experienced 1 -h precipitation $\geq 1 \mathrm{~mm}$ ) in summer (June-August) are collected to further evaluate the TREC, COTREC, and BTREC methods. This period includes a series of convective storms, stratiform storms, and mixed storms. It is noted that the temporal and spatial resolutions of the mosaic image are $12 \mathrm{~min}$ and $0.01^{\circ} \times 0.01^{\circ}$, respectively.

To inspect the improvements of the COTREC and BTREC methods compared to the TREC method, the difference of four verification indexes [the threat score (TS), spatial correlation (COR), the false alarm ratio (FAR), and the probability of detection (POD)] between BTREC or COTREC and TREC are studied. Actually, the TS is the critical success index (CSI; Wilks 2011). The TS, FAR, POD, and CORR are calculated as follows (Wilks 2011; Chen et al. 2017):

$$
\begin{aligned}
\mathrm{TS} & =\frac{n_{s}}{n_{s}+n_{f}+n_{m}}, \\
\mathrm{FAR} & =\frac{n_{f}}{n_{s}+n_{f}}, \\
\mathrm{POD} & =\frac{n_{s}}{n_{s}+n_{m}},
\end{aligned}
$$



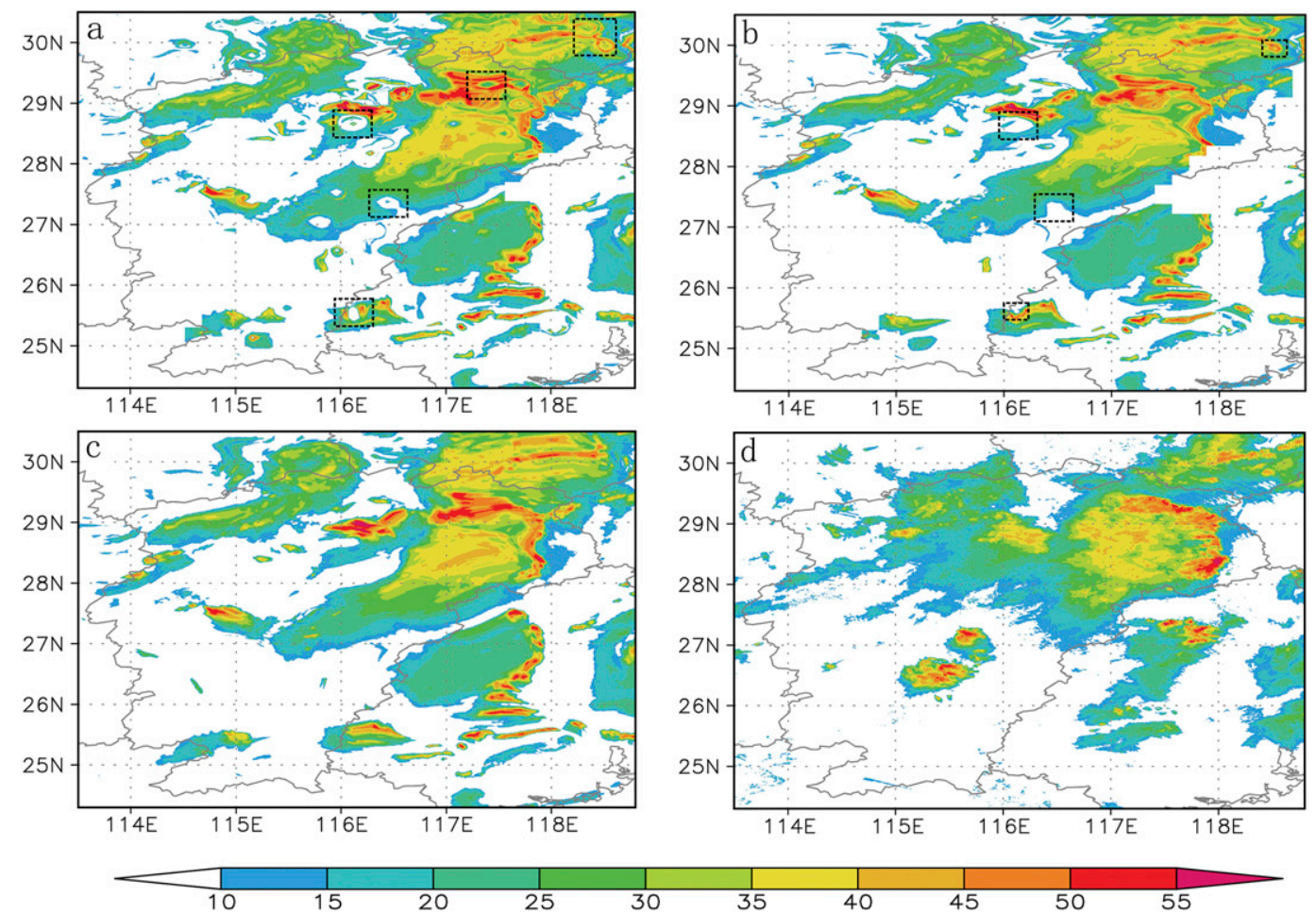

FIG. 9. Composite reflectivity ( $\mathrm{dBZ}$ ) of the 2-h extrapolations based on (a) TREC vectors, (b) COTREC vectors, and (c) BTREC vectors and (d) the observations at 1100 UTC 30 June 2018.

and

$$
\operatorname{CORR}=\frac{\sum_{i=1}^{n} \sum_{j=1}^{m}\left(O_{i, j}-\overline{O_{i, j}}\right)\left(F_{i, j}-\overline{F_{i, j}}\right)}{\left[\sum_{i=1}^{n} \sum_{j=1}^{m}\left(O_{i, j}-\overline{O_{i, j}}\right)^{2}\right]^{1 / 2}\left[\sum_{i=1}^{n} \sum_{j=1}^{m}\left(F_{i, j}-\overline{F_{i, j}}\right)^{2}\right]^{1 / 2}},
$$

where $n_{s}, n_{f}$, and $n_{m}$ are the number of grids for a successful forecast, a false alarm, and a miss within the dashed rectangle in Fig. 1, respectively. Meanwhile, the threshold to identify whether a grid is correctly predicted is set as $10 \mathrm{~dB} Z$. The results were similar when the threshold was set to 20 and $30 \mathrm{~dB} Z$. Variables $O_{i, j}$ and $F_{i, j}$ are the observation and forecast in the grid of $(i, j)$ within the dashed rectangle in Fig. 1 . Selecting the dashed-rectangle area instead of the whole region in Fig. 1 avoids the boundary effect.

Figure 10 shows the mean difference of TS, CORR, FAR and POD between the BTREC (COTREC) and TREC for 1- and 2-h extrapolations. It can be seen from Fig. 10a that for both the COTREC and BTREC, the differences in TS, CORR, and POD are more than 0 , and the difference in FAR is less than 0 . This indicates that the COTREC and BTREC methods improve the 1-h forecast of the TREC method. More importantly, the magnitudes of the difference in the four verification indexes of the BTREC are obviously more than those of the COTREC (Fig. 10a). This statistical analysis further verifies that the performance of BTREC is better than that of COTREC for the 1-h extrapolation. When the extrapolated time is prolonged to $2 \mathrm{~h}$, the mean differences in the four verification indexes of the BTREC method (i.e., red histogram in Fig. 10b) are similar with those for $1 \mathrm{~h}$ (in fact, their magnitudes reduce approximately by $20 \%$ ). However, although the differences in TS, CORR, and POD of the COTREC are still positive and the difference in FAR of the COTREC is still negative, their magnitudes are reduced by about $80 \%$ compared to those for $1 \mathrm{~h}$, closing to 0 (Fig. 10b). This implies that the BTREC method significantly improves the 2-h extrapolation on the basis of the TREC method, while the COTREC brings relatively small improvement.

It is noted that although the mean differences in the four verification indexes for 1- and 2-h extrapolation between BTREC and TREC are small (less than 0.01), they pass the statistical tests on $95 \%$ confidence level. The mean differences in the four verification indexes between COTREC and TREC for 1-h extrapolation are also statistically significant at the $95 \%$ level, but 

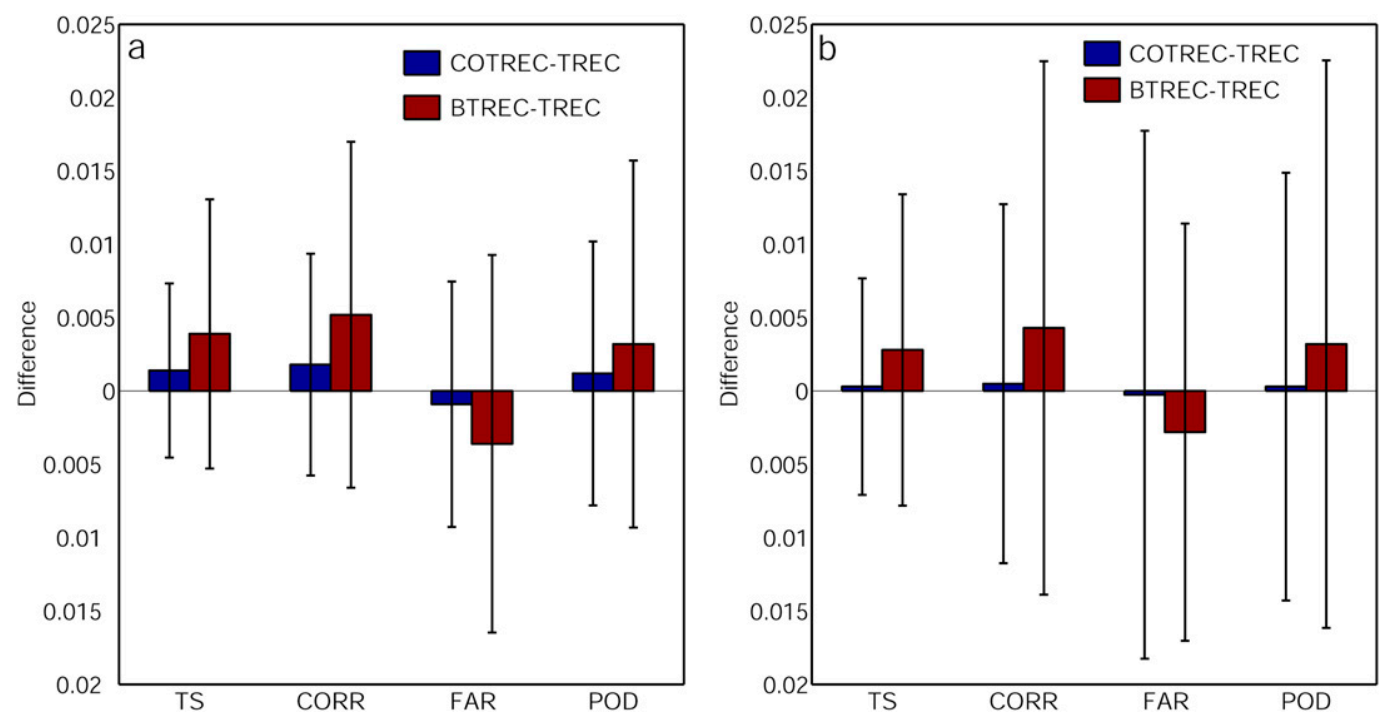

FIG. 10. Mean differences in verification indices between BTREC and TREC and between COTREC and TREC for the (a) 1- and (b) 2-h extrapolations. The error bars represent the standard deviations.

those for 2-h extrapolation are not significant. In fact, the variations of the radar echo pattern are mainly caused by two factors: movement and development. The extrapolation of the radar echo is based on the assumption that the intensity of the radar echo is not changed during its movement (Rinehart and Garvey 1978; Germann and Zawadzki 2002). As a result, the extrapolation methods (including the TREC, COTREC, and BTREC) can only reflect the movement component of the radar echo, and cannot describe its development. However, there are often obvious changes between the current radar echo pattern and that in the next 1 or $2 \mathrm{~h}$ due to the development of storm. These changes can significantly influence the TS, CORR, FAR, and POD scores. Thus, the mean differences in verification indexes between BTREC/COTREC and TREC may be small on account of the fact that they do not consider the development of a storm.

To examine the performances of the BTREC and COTREC methods in more detail, the frequency distributions of the differences (between BTREC and TREC and between COTREC and REC) in TS, CORR, POD, and FAR at different levels are shown in Fig. 11. It can be seen from Fig. 11a that for both COTREC and BTREC methods, the frequency of the TS difference larger than 0.005 is obviously higher than the frequency of the TS difference smaller than -0.005 . More importantly, the frequency of TS difference larger than 0.005 (especially for that larger than 0.01) in BTREC (about $58 \%$ for the 1-h extrapolation and $49 \%$ for the 2 -h extrapolation) is remarkably higher than that in COTREC (about $40 \%$ for the $1-\mathrm{h}$ extrapolation and $35 \%$ for the 2-h extrapolation), while the frequency of TS difference smaller than -0.005 is similar between COTREC and BTREC (Fig. 11a). The frequency distributions of CORR and POD difference are similar to that of the TS difference (Figs. 11b,c). On the contrary, the frequency of the FAR difference less than -0.005 is obviously higher than the frequency of the FAR difference more than 0.005 (Fig. 11d). The frequency of FAR difference smaller than -0.005 in BTREC is markedly higher than that in COTREC, while the frequency of more than 0.005 is similar with COTREC (Fig. 11d). This evidence verifies that COTREC and BTREC perform better than TREC, and more prominently, the BTREC method can obtain more accurate 1- and 2-h extrapolations than the COTREC method. It is noted that the negative TS, CORR, and POD differences and the positive FAR differences are also apparent in Fig. 11. This is mainly because the real radar echoes usually include some small-scale features whose predictability is less than $2 \mathrm{~h}$ or even less than $1 \mathrm{~h}$.

\section{Conclusions}

In this paper, a method termed BTREC for obtaining the motion of radar echo patterns was proposed. This method is an extension of TREC and uses the Barnes filter to correct the noise and inconsistencies in the TREC vectors. The performance of the BTREC method is tested with real radar data in Jiangxi, China.

Due to the impacts of nonmeteorological echo, shielding, and rapid morphological changes of the radar patterns, the TREC vectors often show inconsistent and 

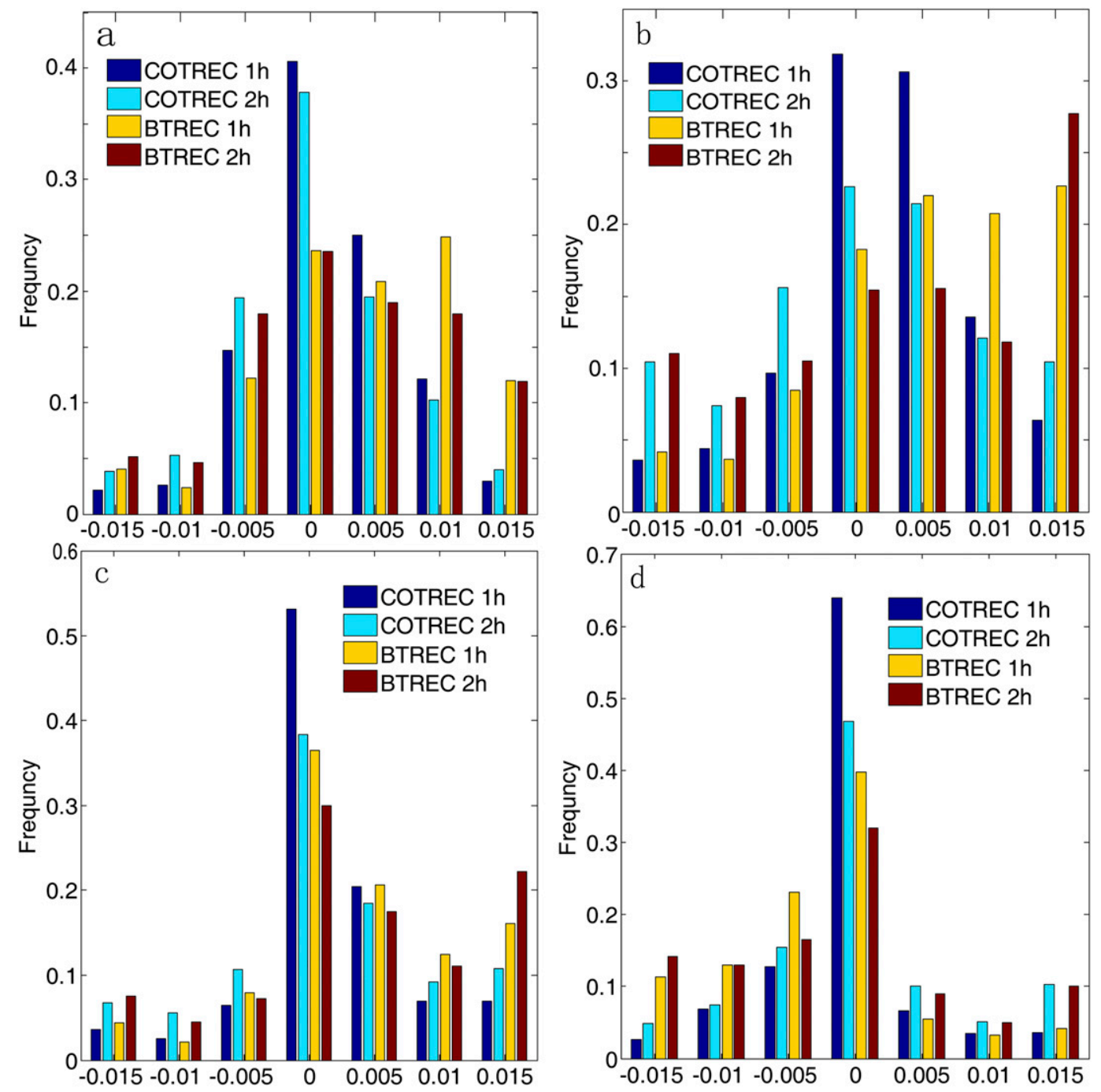

FIG. 11. Histograms of the differences between BTREC and TREC and between COTREC and TREC for (a) TS, (b) CORR, (c) POD, and (d) FAR.

noisy behaviors. The BTREC method was designed to correct these inconsistent and noisy behaviors. Comparative analysis of TREC, COTREC, and BTREC vectors has shown that the COTREC and BTREC methods can effectively smooth the noise and inconsistencies in TREC vectors and the BTREC method can smooth them more thoroughly. As a result, the BTREC method further reduces the divergence and vorticity of the motion vectors.

The BTREC method was applied to extrapolate radar echo patterns over Jiangxi for two cases. Results showed that out of the three methods (BTREC, COTREC, and TREC), the BTREC method performed the best forecasting the radar echo pattern 1 and $2 \mathrm{~h}$ in the future, obviously reducing the distortions in the extrapolated radar echo patterns. To further confirm the performance of the BTREC method, 892 radar composite reflectivity mosaic images with precipitation (of about 2500 stations of Jiangxi Province, more than 10 stations have experienced 1 -h precipitation $\geq 1 \mathrm{~mm}$ ) in summer (June-August) over Jiangxi were collected to test the three methods. The results showed that in the 1-h extrapolation, the mean differences in TS, CORR, and POD between BTREC or COTREC and TREC were more than 0 , while the mean difference in FAR was less than 0, implying the better performance of COTREC and BTREC compared with TREC. Moreover, the magnitudes of the mean differences in TS, CORR, FAR, and POD of BTREC were more than those of COTREC, indicating that BTREC can obtain a more accurate forecast compared to COTREC. When the extrapolated time was prolonged to $2 \mathrm{~h}$, the mean 
differences in TS, CORR, FAR, and POD of the BTREC method were similar to those of 1-h extrapolation. Although the positive and negative signs of mean differences in TS, CORR, FAR, and POD of COTREC were still the same as those of 1-h extrapolation, their magnitudes were significantly reduced by about $80 \%$ (close to 0 ). Also, these mean differences for the 2-h extrapolation of COTREC were not statistically significant at the $95 \%$ level. This implies that the BTREC method still has a significant improvement in the 2-h extrapolation, while the improvement of the COTREC method is not significant.

Although the BTREC method proposed in this paper has improved the extrapolation of the radar echo pattern, the parameter $C$ in the Barnes filter (i.e., the cutoff wavelength) is closely related to the forecast time length. Therefore, future work will focus on the relationship of the parameter $C$ and the forecast time length. In addition, the BTREC method is similar to the TREC and COTREC methods and does not consider the physical mechanism of a storm's development. It is clear that there is still some possible improvement for the extrapolated algorithm of the radar echo pattern. In particular, the topography, mesoscale environment, surface heat flux, etc., should be introduced to jointly improve the extrapolated algorithm.

Acknowledgments. This study is jointly supported by Jiangxi Provincial Department of science and technology project (Grant 20171BBG70004) and the National Natural Science Foundation of China (Grants 41865003 and 41765001). We thank two anonymous reviewers and the editor for constructive comments that help to improve this manuscript.

\section{REFERENCES}

Askelson, M. A., J.-P. Aubagnac, and J. M. Straka, 2000: An adaptation of the Barnes filter applied to the objective analysis of radar data. Mon. Wea. Rev., 128, 3050-3082, https://doi.org/ 10.1175/1520-0493(2000)128<3050:AAOTBF $>2.0 . C O ; 2$.

Ballard, S. P., Z. Li, D. Simonin, and J.-F. Caron, 2016: Performance of 4D-Var NWP-based nowcasting of precipitation at the Met Office for summer 2012. Quart. J. Roy. Meteor. Soc., 142, 472-487, https://doi.org/10.1002/qj.2665.

Barker, D. M., W. Huang, Y. R. Guo, and Q. N. Xiao, 2004: A three-dimensional (3DVAR) data assimilation system for use with MM5: Implementation and initial results. Mon. Wea. Rev., 132, 897-914, https://doi.org/10.1175/1520-0493(2004) 132<0897:ATVDAS > 2.0.CO;2.

Barnes, S. L., 1964: A technique for maximizing details in numerical weather map analysis. J. Appl. Meteor., 3, 396-409, https:// doi.org/10.1175/1520-0450(1964)003<0396:ATFMDI > 2.0.CO;2. 1973: Mesoscale objective map analysis using weighted time series observations. NOAA Tech. Memo. ERL NSSL-62, 60 pp., https://repository.library.noaa.gov/view/noaa/17647.
Bechini, R., and V. Chandrasekar, 2017: An enhanced optical flow technique for radar nowcasting of precipitation and winds. J. Atmos. Oceanic Technol., 34, 2637-2658, https://doi.org/ 10.1175/JTECH-D-17-0110.1.

Bertsekas, D. P., 1982: Constrained Optimization and Lagrange Multiplier Methods. Academic Press, 395 pp.

Chen, L., J. H. Dai, and L. Tao, 2009: Application of an improved TREC algorithm (COTREC) for precipitation nowcast (in Chinese). J. Trop. Meteor., 25 (1), 117-122.

Chen, M., and Coauthors, 2010: Introduction of Auto-nowcasting system for convective storm and its performance in Beijing Olympics meteorological service (in Chinese). J. Appl. Meteor. Sci., 21 (4), 395-403.

Chen, Y., H. Lan, X. Chen, and W. Zhang, 2017: A nowcasting technique based on application of the particle filter blending algorithm. J. Meteor. Res., 31, 931-945, https://doi.org/10.1007/ s13351-017-6557-9.

Dixon, M., and G. Wiener, 1993: TITAN: Thunderstorm Identification, Tracking, and Nowcasting - A radar-based methodology. J. Atmos. Oceanic Technol., 10, 785-797, https://doi.org/ 10.1175/1520-0426(1993)010<0785:TTITAA $>2.0$. CO;2.

Doswell, C. A., 1977: Obtaining meteorologically significant surface divergence fields through the filtering property of objective analysis. Mon. Wea. Rev., 105, 885-892, https://doi.org/ 10.1175/1520-0493(1977)105<0885:OMSSDF $>2.0$. CO; 2 .

Duchon, C. E., 1979: Lanczos filtering in one and two dimensions. J. Appl. Meteor., 18, 1016-1022, https://doi.org/10.1175/ 1520-0450(1979)018<1016:LFIOAT > 2.0.CO;2.

Evans, J. E., and E. R. Ducot, 1994: The Integrated Terminal Weather System (ITWS). Lincoln Lab. J., 7 (192), 449-474.

Germann, U., and I. Zawadzki, 2002: Scale-dependence of the predictability of precipitation from continental radar images. Part I: Description of the methodology. Mon. Wea. Rev., 130, 2859-2873, https://doi.org/10.1175/1520-0493(2002)130<2859: $\mathrm{SDOTPO}>2.0 . \mathrm{CO} ; 2$.

Golding, B. W., 1998: Nimrod: A system generating automated very short range forecasts. Meteor. Appl., 5, 1-16, https:// doi.org/10.1017/S1350482798000577.

Gomis, D., and S. Alonso, 1990: Diagnosis of a cyclogenetic event in the western Mediterranean using an objective technique for scale separation. Mon. Wea. Rev., 118, 723-736, https://doi.org/ 10.1175/1520-0493(1990)118<0723:DOACEI $>2.0 . C O ; 2$.

Hu, S., C. Luo, X. Huang, H. Li, and R. He, 2012: Comparisons of one hour precipitation forecast between the radar extrapolation and mesoscale numerical model (in Chinese). Meteor. Mon., 38 (3), 274-280.

Huang, X., K. Zhu, and K. Zhao, 2017: The improved TREC algorithm and its application to the precipitation nowcasting for landfall typhoon (in Chinese). J. Meteor Sci., 37 (5), 610-618.

Hwang, Y., A. J. Clark, V. Lakshmanan, and S. E. Koch, 2015: Improved nowcasts by blending extrapolation and model forecasts. Wea. Forecasting, 30, 1201-1217, https://doi.org/ 10.1175/WAF-D-15-0057.1.

Johnson, J. T., P. L. MacKeen, A. Witt, E. D. Mitchell, G. J. Stumpf, M. D. Eilts, and K. W. Thomas, 1998: The storm cell identification and tracking algorithm: An enhanced WSR-88D algorithm. Wea. Forecasting, 13, 263-276, https://doi.org/10.1175/ 1520-0434(1998)013<0263:TSCIAT >2.0.CO;2.

Kyznarová, H., and P. Novák, 2009: CELL TRACK-Convective cell tracking algorithm and its use for deriving life cycle characteristics. Atmos. Res., 93, 317-327, https://doi.org/10.1016/ j.atmosres.2008.09.019. 
Li, L., W. Schmid, and J. Joss, 1995: Nowcasting of motion and growth of precipitation with radar over a complex orography. J. Appl. Meteor., 34, 1286-1300, https://doi.org/10.1175/ 1520-0450(1995)034<1286:NOMAGO >2.0.CO;2.

Liang, Q., Y. Feng, W. Deng, S. Hu, Y. Huang, Q. Zeng, and Z. Chen, 2010: A composite approach of radar echo extrapolation based on TREC vectors in combination with modelpredicted winds. Adv. Atmos. Sci., 27, 1119-1130, https:// doi.org/10.1007/s00376-009-9093-4.

Lin, C., S. Vasic, A. Kilambi, B. Turner, and I. Zawadzki, 2005: Precipitation forecast skill of numerical weather prediction models and radar nowcasts. Geophys. Res. Lett., 32, L14801, https://doi.org/10.1029/2005GL023451.

Maddox, R. A., 1980: An objective technique for separating macroscale and mesoscale features in meteorological data. Mon. Wea. Rev., 108, 1108-1121, https://doi.org/10.1175/ 1520-0493(1980)108<1108:AOTFSM > 2.0.CO;2.

Mandapaka, P. V., U. Germann, L. Panziera, and A. Hering, 2012: Can Lagrangian extrapolation of radar fields be used for precipitation nowcasting over complex alpine orography? Wea. Forecasting, 27, 28-49, https://doi.org/10.1175/ WAF-D-11-00050.1.

Morris, W. E., and P. J. Smith, 2001: Cyclolysis: A diagnosis of two extratropical cyclones. Mon. Wea. Rev., 129, 2714, https:// doi.org/10.1175/1520-0493(2001)129<2714:CADOTE >2.0.CO;2.

Mueller, C., T. Saxen, R. Roberts, J. Wilson, T. Betancourt, S. Dettling, N. Oien, and J. Yee, 2003: NCAR Auto-Nowcast System. Wea. Forecasting, 18, 545-561, https://doi.org/10.1175/ 1520-0434(2003)018<0545:NAS $>2.0$. CO;2.

Otsuka, S., and Coauthors, 2016: Precipitation nowcasting with three-dimensional space-time extrapolation of dense and frequent phased-array weather radar observations. Wea. Forecasting, 31, 329-340, https://doi.org/10.1175/WAF-D-15-0063.1.

Overton, K. J., and T. E. Weymouth, 1979: A noise reducing preprocessing algorithm. Proc. IEEE Computer Science Conference on Pattern Recognition and Image Processing, Chicago, IL, IEEE, 498-507.

Press, W. H., B. P. Flannery, S. A. Teukolsky, and W. T. Vetterling, 1991: Numerical Recipes: The Art of Scientific Computing. Cambridge University Press, 702 pp.

Ray, P. S., 1980: Single- and multiple-Doppler radar observations of tornadic storms. Mon. Wea. Rev., 108, 1607-1625, https:// doi.org/10.1175/1520-0493(1980)108<1607:SAMDRO>2.0.CO;2.

Rinehart, R. E., 1981: A pattern recognition technique for use with conventional weather radar to determine internal storm motions. Atmos. Technol., 13, 119-134.
— , and E. T. Garvey, 1978: Three-dimensional storm motion detection by conventional weather radar. Nature, 273, 287289, https://doi.org/10.1038/273287a0.

Sasaki, Y., 1958: An objective analysis based on the variational method. J. Meteor. Soc. Japan, 36, 77-88, https://doi.org/ 10.2151/jmsj1923.36.3_77.

- , 1970: Some basic formalisms in numerical variational analysis. Mon. Wea. Rev., 98, 875-883, https://doi.org/10.1175/ 1520-0493(1970)098<0875:SBFINV > 2.3.CO;2.

Seed, A. W., C. E. Pierce, and K. Norman, 2013: Formulation and evaluation of a scale decomposition-based stochastic precipitation nowcast scheme. Water Resour. Res., 49, 6624-6641, https://doi.org/10.1002/wrcr.20536.

Wilks, D. S., 2011: Statistical Methods in the Atmospheric Sciences. 3rd ed. International Geophysics Series, Vol. 100, Academic Press, $704 \mathrm{pp}$.

Wong, M. C., 2006: From SWIRLS to RAPIDS: Nowcast applications development in Hong Kong. WMO PWS Workshop on Warnings of Real-Time Hazards by Using Nowcasting Technology, Sydney, Australia, WMO, 20 pp., https:// www.wmo.int/pages/prog/amp/pwsp/documents/Wong SWIRLStoRAPIDS.pdf.

Woo, W., and W. Wong, 2017: Operational application of optical flow techniques to radar-based rainfall nowcasting. Atmosphere, 8, 48, https://doi.org/10.3390/atmos8030048.

Wu, W., H. Zou, J. Shan, and S. Wu, 2018: A dynamical $Z-R$ relationship for precipitation estimation based on radar echotop height classification. Adv. Meteor., 2018, 8202031, https:// doi.org/10.1155/2018/8202031.

Zhang, H., G. Li, and S. Wang, 2014: Mesoscale filtering analysis of a regional heavy rain by southwest vortex (in Chinese). Plateau Meteor., 33 (2), 361-371.

Zhao, Y., and Coauthors, 2007: Weather radar echo image processing based on median filter and wavelet transform (in Chinese). Sci. Meteor. Sin., 27, 63-68.

Zou, H., J. Shan, and S. Deng, 2014: Study of the Doppler radar data gridding (in Chinese). Meteor. Disaster Reduct. Res., 37 (2), 23-30

- S. Wu, X. Yi, and N. Wu, 2018a: The Application of Barnes filter to positioning the center of landed tropical cyclone in numerical models. Adv. Meteor., 2018, 1747530, https:// doi.org/10.1155/2018/1747530.

, S. Zhang, X. Liang, and X. Yi, 2018b: Improvement in the methods for removing isolated non-meteorological echoes and ground clutter in CINRAD. J. Meteor. Res., 32, 584-597, https://doi.org/10.1007/s13351-018-7176-9. 